\title{
PRINCIPAL SLOPE ESTIMATION AT SAR BUILDING LAYOVERS
}

\author{
Cristian Rossi, Michael Eineder, Sergi Duque, Thomas Fritz and Alessandro Parizzi \\ German Aerospace Center (DLR), Remote Sensing Technology Institute, Wessling, Germany.
}

\begin{abstract}
Spectral estimation is considered in the paper as an additional instrument towards a better understanding of the physical phenomena behind the layover scattering decomposition. A super-resolution technique is employed to derive the fringe frequencies characterizing the layover portion. Due to the limited estimation support, only the dominant frequency is found to be reliable information. The non-linear relationship with slopes is employed to derive a principal slope map. A bistatic interferometric scenario is tested. It is found that for the majority of the detections the facade contribution is the prevailing one due to the presence of targets with a high backscattered signal return at the vertical slope. The number of layover contributors is assessed prior to the spectral estimation. It has been estimated that the signal return is dominated by a single contribution for the majority of the layovers.
\end{abstract}

Index Terms - layover, InSAR, urban areas, MUSIC

\section{INTRODUCTION}

The mapping of urban areas with SAR sensors is increasing his attractiveness due to the increment of high resolution data available to science users. With new missions, as TanDEM-X, and expected future ones, urban SAR research is growing in a field dominated nowadays by optical or LiDAR sensors [1]. The bistatic interferometric data quality has been reported capable to allow the detection of the layover portion of buildings. In [2], the focus was on the interferometric processor, and in particular on the geocoding stage. The peculiar layover phase trend has been exploited in the mapping matrices in a way to precisely identify layovers without a-priori hypotheses and without employing external high-resolution DEMs.

Due to the complexity of the urban environment, it is not possible to easily provide radar cross section estimation for the different layover contributors. Too many aspects play a role as ground surface roughness, building material, number of bounces, roof tilt and so on. Every building should be then treated singularly for the radar scattering decomposition. For instance, the simplest building shape is a rectangular cuboid, with the ground, roof and wall respectively represented by the lower and higher horizontal and the vertical segments (Fig. 1). In the layover area the signal return is a superposition of these contribution. The objective of this paper is to exploit

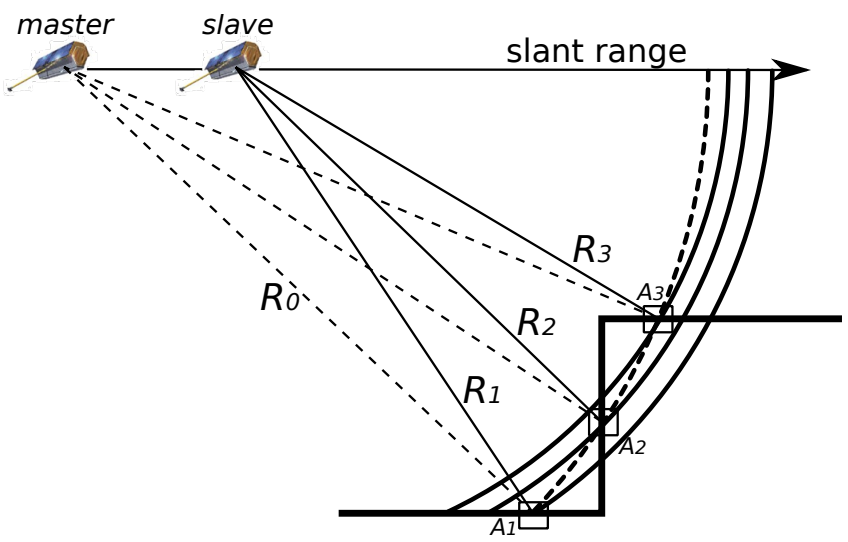

Fig. 1. Interferometric signal model for a building layover pixel. $R_{1}$ is the slant range distance between the satellite and the ground scatterer $A_{1}$, similarly for the wall scatterer $\left(R_{2}\right.$, $\left.A_{2}\right)$ and the roof scatterer $\left(R_{3}, A_{3}\right) . R_{0}$ is the master distance between the satellite and the three scatterers.

the interferometric phase in order to derive the principal contributor and to provide an estimation of the weight of other contributors. In this context, the fringe frequency estimation can be helpful and is investigated in the next section.

\section{PRINCIPAL SLOPE ESTIMATION}

The local slope $\alpha$ is linked to the range fringe frequency $f_{r g}$, as in the spectral shift theory [3]:

$$
f_{r g}=-\frac{f_{0} \Delta \theta}{\tan (\theta-\alpha)},
$$

where $\Delta \theta=B_{n} / R_{0}$, being $B_{n}$ the normal baseline between the satellites, $f_{0}$ the carrier frequency and $\theta$ the master looking angle. Thus, for the simple building model in Fig. 1, the frequency corresponding to the ground $f_{\text {ground }}$ is equal to the roof one $f_{\text {roof }}$ since they have the same slope. In case of two fringe frequencies as for the cuboid model, a relationship between the ground and wall frequencies is established. (1) is rewritten introducing the local incidence angle $\theta_{\text {inc }}$ :

$$
f_{r g}=-\frac{f_{0} \Delta \theta}{\tan \left(\theta_{i n c}\right)} .
$$


Due to the 90 deg separation,

$$
\theta_{\text {inc }}^{\text {ground }}=-\left(\frac{\pi}{2}-\theta_{\text {inc }}^{\text {wall }}\right) .
$$

In the frequency domain (3) corresponds to a negative frequency for the ground/roof and a positive one for the wall (in the convention of (2). The frequencies result:

$$
\begin{aligned}
f_{\text {ground }} & =-\frac{f_{0} \Delta \theta}{\tan \left(\theta_{\text {inc }}\right)} \\
f_{\text {wall }} & =-\frac{f_{0} \Delta \theta}{\tan \left(\theta_{\text {inc }}-\frac{\pi}{2}\right)}=f_{0} \Delta \theta \tan \left(\theta_{\text {inc }}\right) \\
f_{\text {wall }} & =-f_{\text {ground }} \tan ^{2}\left(\theta_{\text {inc }}\right)
\end{aligned}
$$

The frequencies derived in (4) characterising the layover area can be estimated directly from the interferogram. MUSIC, a classical super-resolution estimation method, is analysed in the paper. It is based on the maximization of the pseudo-spectrum, computed using estimates of the eigenvectors of the correlation matrix associated with the input layover samples [4]. The estimation is performed for every detected building layover, assuming each slant range layover portion as an independent realization of the same process. This assumption implies the preservation of the building slopes in the azimuth direction, thus presupposing a building modelled by planar surfaces. To be noticed, with this assumption the building orientation is not impacting in the estimation. Nevertheless, a complexity comes from the varying range support. In fact, in contrast to a standard frequency estimation, which usually assumes a constant size for each realization, for layover areas the realizations dimension may vary. Using a weight for each realization depending on the realization size circumvents this issue. We define valid realization a realization whose size exceeds a minimum value. The correlation matrix is estimated with a spatial smoothing method [4] in order to use all the sample of valid realizations. Naming $n_{\text {lay }}$ the realization dimension and $p$ the correlation matrix order, the weight is defined as $\left(n_{\text {lay }}+p-1\right) /\left(n_{\text {lay }}^{\max }+p-1\right)$, where $n_{\text {lay }}^{\max }$ is the largest realization size.

Once estimated the frequencies, the related ground slopes can be derived inverting (1):

$$
\alpha=\arctan \left(\frac{f_{r g} \tan \theta+f_{0} \Delta \theta}{f_{r g}-f_{0} \Delta \theta \tan \theta}\right) .
$$

Due to the non linearity of (5), the estimation accuracy at different slopes varies significantly. For example, an error of $0.4 \mathrm{Mhz}$ in the frequency estimation for $90 \mathrm{deg}$ yields an error of about $2 \mathrm{deg}$, whereas the same inaccuracy for $35 \mathrm{deg}$ brings an estimation error of only $0.07 \mathrm{deg}$.

A simulation with the TanDEM-X system parameters of the test case investigated in the next section is performed to evaluate the minimum range support for four different SNR levels $(0,5,10,15 \mathrm{~dB})$ (Table 1). A stronger weight is chosen for a tone (wall in this case) to assess a weak tone detection accuracy.

Table 1. THE MINIMUM RANGE SUPPORT $n_{l a y}^{m i n}$ FOR A 80\% WALL WEIGHT AND A 20\% GROUND WEIGHT.

\begin{tabular}{ccc}
\hline & wall $^{\text {a }}$ & ground/roof $^{\text {a }}$ \\
\hline MUSIC| $\left.\right|_{S N R=15 d B}$ & $12 / 14 / 17$ & $18 / 32 / 37$ \\
MUSIC| & $14 / 17 / 23$ & $32 / 37 / 43$ \\
MUSIC $\left.\right|_{S N R=5 d B}$ & $15 / 18 / 35$ & $38 / 42 / 60$ \\
MUSIC| $\left.\right|_{S N R=0 d B}$ & $35 \% / 37 / 41$ & $62 \% 75 \%-$ \\
\hline
\end{tabular}

\footnotetext{
${ }^{\mathrm{a}}$ Support for an absolute error of $0.8 / 0.4 / 0.2 \mathrm{MHz}$.

$\mathrm{b}$ The curve is not monotonous. This value refers to the worst case.
}

Considering the simulation and the limited estimation support of building layovers, only the dominant slope is derived. Accurate secondary slopes estimations require on average a large support.

\section{RESULTS}

The super resolution algorithm is tested in a real interferometric TanDEM-X scenario. A bistatic spotlight acquisition acquired on the 4th of January, 2012 over the city of Berlin (Germany) is chosen. The satellites had a normal baseline of about 110 meters yielding a height of ambiguity of 65 meters, with the incidence angle at the center of the scene being 41.8 degrees. The same dataset was used in [1] to test the TanDEM-X DEM generation capabilities over urban areas. As the paper purpose is to investigate over single buildings, a spotlight acquisition is of fundamental importance due to the high resolution capable to isolate building signatures. The bistatic configuration is as well an advantage to avoid false detection resulting from temporal decorrelations.

The principal slope, derived as in (5) by employing the conventional MUSIC algorithm for the frequency estimation, is shown in Fig. 2. Considering the performed simulations, the fringe frequency is estimated for layover patches having a minimum range and azimuth support of 15 and 10 samples, respectively. The covariance matrix order is adaptively chosen depending on the actual range support. In particular, the order is defined as the minimum common support exceeding the minimum valid realisation. The model order is estimated in the processing by adopting the Minimum Description Length (MDL) [4] algorithm and fixing three tones as maximum order. A complementary map, with the number of frequencies in the area, representing different slopes and then contributing to the total scattered field, is also automatically generated (not shown here).

An analysis of the result indicates that for about $60 \%$ of the analyzed buildings (in total 866) wall has the dominance in the signal return. Flat roofs (or ground) dominates for about $25 \%$ and other slopes, as tilted roofs, for the remaining $15 \%$. 
principal slope [deg]

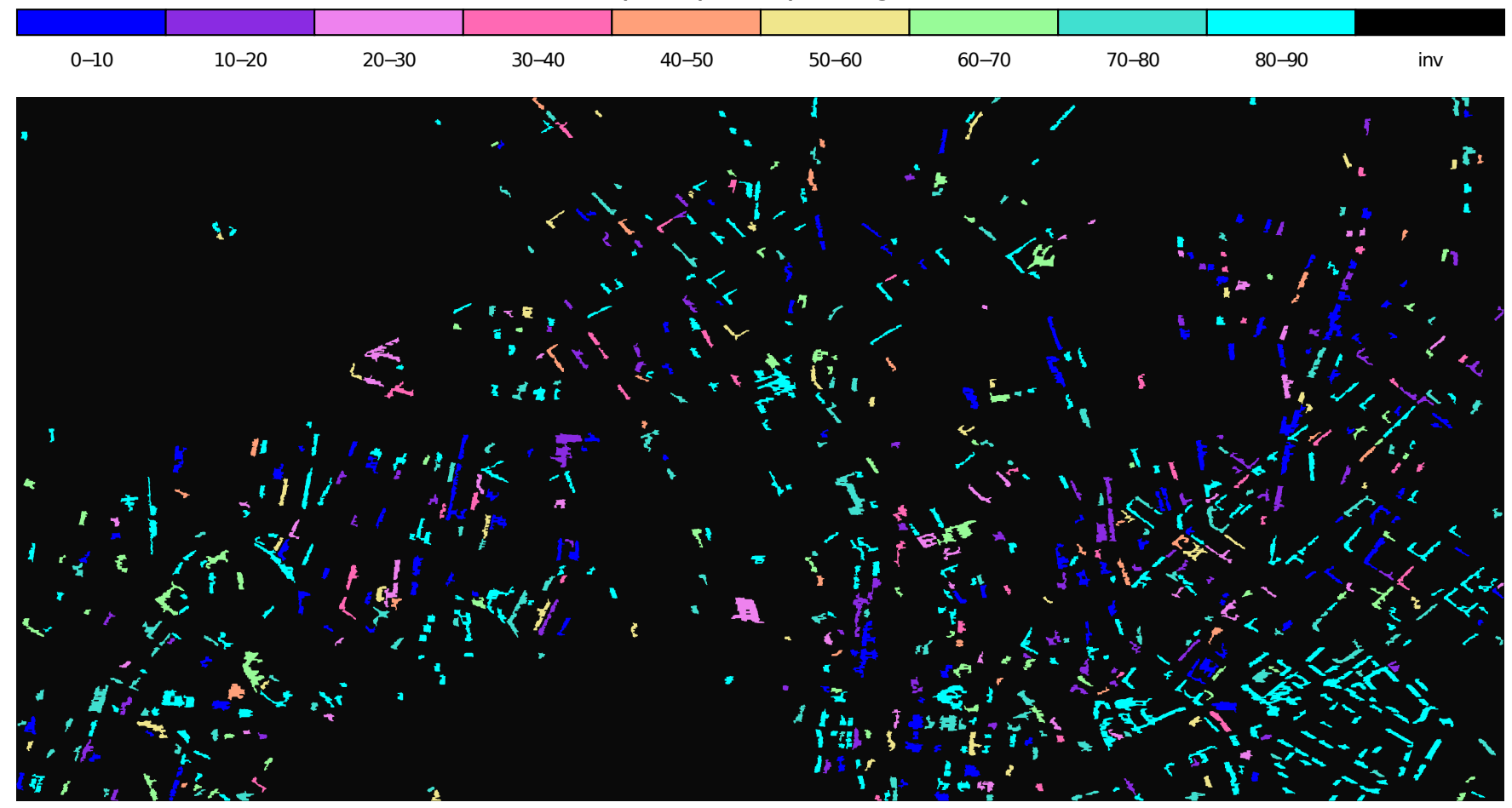

Fig. 2. Principal slope for the detected buildings. A segmentation on the detected map and a conventional MUSIC algorithm is used to detect the dominant frequency for detected buildings having a minimum range and azimuth support of 15 and 10 samples. The color scale is at the top.

A first consideration is about the generally larger wall support at the acquisition incidence angle due to the buildings conformation of the city under analysis. In fact, excluding wall portions not visible by the radar due to local occlusion (e.g. trees or close buildings), the wall is generally totally included in the portion whereas roofs are only summing up for a section of the total layover support since their extension is smaller than the walls one. A second consideration is about the balconies-windows configuration, which creates a set of strong reflectors at the vertical slope. For these configurations, the facade layover contribution dominates over the others. An example is provided in Fig. 3. The mentioned buildings conformation is evident for this portion, representing the southern-eastern part of the derived map. The derived spectrum and pseudo-spectrum for a benchmark structure are plotted at the bottom of the figure. A first degree model is detected. The conventional MUSIC algorithm estimates a principal slope close to 90 degrees. On the contrary, the FFT result - also tested here - is not accurately detecting the wall frequency. Generally, FFT results are much more sensible to local backscattering variations. The same spectral features are encountered for all the buildings in the area, and a vertical dominance is estimated as visible in the bottom-right part of the result in Fig. 2. Strong reflectors at the roof tops makes the roof slope dominate. On the whole, it has been experimentally verified that when various scatterers at a specific facet present an high backscattered signal return, their facet

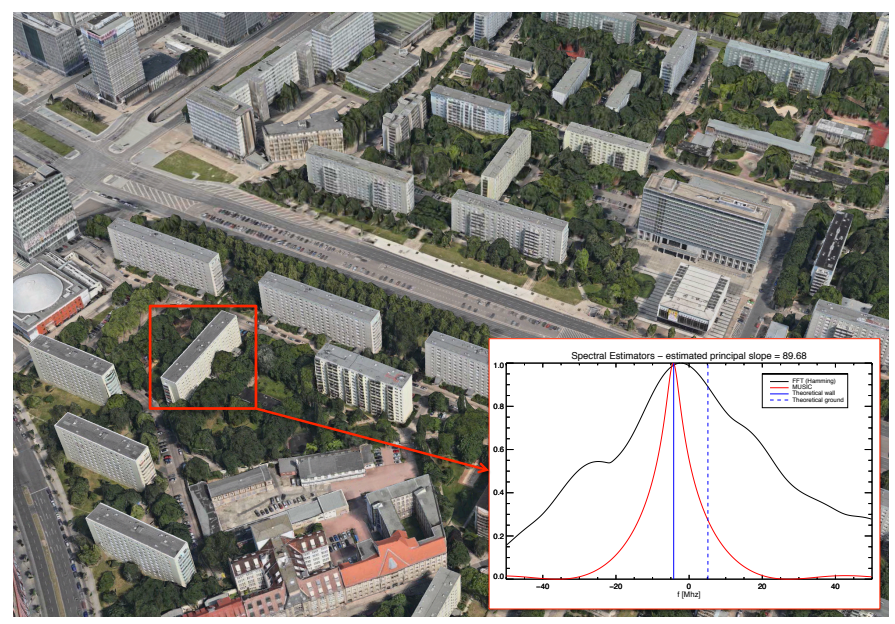

Fig. 3. Three dimensional view of the southern-eastern part of the map (Apple Maps (C)). An exemplary estimation result is highlighted at the bottom. 
slope is measured and a single slope is estimated. The presence of strong scatterers at different facets complicates the situation. In these cases, multiple slopes are estimated. The study in Section II, presenting one of the unlimited possible signal interactions in the layover area, demonstrated the difficulties in accurately estimating secondary slopes due to the limited estimation support. Following this analysis, it is possible to generalize that the expected estimated slope accuracy is higher when a single layover contributor is detected.

\section{CONCLUSIONS}

The main message of the paper is to consider spectral estimation as an additional instrument towards a better understanding of the physical phenomena behind the layover scattering decomposition. In the urban remote sensing framework, the use of bistatic interferometry reveals its potential not only by allowing the generation of high resolution DEMs but also in deriving building layover maps with overlaid physical parameters estimation using a single acquisition.

\section{REFERENCES}

[1] C. Rossi and S. Gernhardt, "Urban DEM Generation, Analysis and Enhancements using TanDEM-X," ISPRS Journal of Photogrammetry and Remote Sensing, vol. 85, pp. 120131, 2013.

[2] C. Rossi,T. Fritz and M. Eineder, "Detecting Building Layovers in a SAR Interferometric Processor Without External References," in 10th European Conference on Synthetic Aperture Radar (EUSAR14).

[3] F. Gatelli, A. Monti Guarnieri, F. Parizzi, P. Pasquali, C. Prati and F. Rocca, "The wavenumber shift in SAR interferometry," IEEE Trans. Geosci. Remote Sens., vol. 32, no. 4, pp. 855-865, Jul. 1994.

[4] S. M. Kay, Modern Spectral Estimation: Theory and Application. En- glewood Cliffs, NJ: Prentice-Hall, 1988. 